\title{
Decomposition and suboptimal control in dynamical systems
}

\author{
Felix L. Chernousko \\ Institute for Problems in Mechanics of the Russian Academy of Sciences \\ pr. Vernadskogo 101, Moscow 117526, Russia.
}

Tel: 007-095-4340207. Fax: 007-095-9382048.

e-mail: chern@ipm.msk.su

\begin{abstract}
Constructive methods of control for dynamical systems subject to bounded controls are proposed. Lagrangian systems governed by nonlinear ordinary differential equations and linear systems governed by partial differential equations are considered. The approach is based on the decomposition of the original system with a finite or infinite number of degrees of freedom into simple subsystems with one degree of freedom each. Methods of optimal control and differential games are applied to the subsystems. Explicit feedback control laws are obtained which bring the original dynamical systems to the prescribed terminal states in finite time. Upper estimates on the control time are obtained. Controllability of systems subject to bounded controls is discussed. The proposed methods are applicable to robots and elastic systems.
\end{abstract}

\section{Keywords}

Decomposition, nonlinear dynamical systems, optimal control, differential games, controllability

\section{INTRODUCTION}

The well known classical methods of automatic control present the control $u$ as a linear operator of the state $x: u=L x$. Thus, in the vicinity of the terminal state $x=0$, the control $u$ becomes small: $u \rightarrow 0$. Here, not all control possibilities are used, and the control time is infinite: $x \rightarrow 0$ as $t \rightarrow \infty$. On the other hand, far away from the terminal state, the control $u$ becomes large enough and may violate constraints usually imposed on control.

Methods of optimal control (Pontryagin et al, 1972) are free from these drawbacks, take account of control constraints and can bring the system to the terminal state in minimal time. However, it is very difficult to obtain optimal feedback controls for nonlinear systems.

In this paper, we propose a constructive approach for obtaining bounded controls in dynamical systems. We consider systems of two types: nonlinear systems described by the Lagrangian ordinary differential equations and linear systems described by partial differential equations. To obtain the control laws, we use the decomposition of our systems 
with a finite or infinite number of degrees of freedom into simple subsystems with one degree of freedom each. After that, methods of optimal control and differential games are applied to the subsystems. Explicit feedback control laws are given. The results presented here were obtained in (Chernousko, 1990, 1992a, 1992b, 1993a, 1993b, Ananyevskii et al, 1995) where full proofs, upper estimates on the total time of motion, more details and examples can be found.

\section{LAGRANGIAN SYSTEMS}

Consider a nonlinear dynamical system governed by Lagrange's equations

$\frac{d}{d t} \frac{\partial T}{\partial \dot{q}_{k}}-\frac{\partial T}{\partial q_{k}}=Q_{k}+F_{k}(q, \dot{q}, t), \quad i=1, \ldots, n$.

Here $t$ is time, $\boldsymbol{q}=\left(q_{1}, \ldots, q_{n}\right)$ is an $n$-vector of generalized coordinates, $T$ is the kinetic energy, $Q_{k}$ are control forces subject to the constraints

$\left|Q_{k}\right| \leq Q_{k}^{0}, \quad k=1, \ldots, n$

where $Q_{k}^{0}$ are given constants, $F_{k}$ are all other forces including uncertain disturbances. The kinetic energy of the system $T$ is given by

$T=\frac{1}{2}(\boldsymbol{A}(\boldsymbol{q}) \dot{\boldsymbol{q}}, \dot{\boldsymbol{q}})=\frac{1}{2} \sum_{i, j=1}^{n} A_{i j}(\boldsymbol{q}) \dot{q}_{i} \dot{q}_{j}$

where $\boldsymbol{A}(\boldsymbol{q})$ is a symmetrical positive definite $n \times n$ - matrix, and $(\cdot, \cdot)$ denotes a scalar product of vectors. Substituting (3) into (1) we obtain

$\boldsymbol{A}(\boldsymbol{q}) \ddot{q}=\boldsymbol{Q}+\boldsymbol{F}(\boldsymbol{q}, \dot{\boldsymbol{q}}, t)+\boldsymbol{S}(\boldsymbol{q}, \dot{\boldsymbol{q}})$

where the following notation is introduced

$\boldsymbol{Q}=\left(Q_{1}, \ldots, Q_{n}\right), \quad \boldsymbol{F}=\left(F_{1}, \ldots, F_{n}\right) ; \quad \boldsymbol{S}=\left(S_{1}, \ldots, S_{n}\right)$,

$S_{k}(\boldsymbol{q}, \dot{\boldsymbol{q}})=\sum_{i, j=1}^{n} \Gamma_{k i j}(\boldsymbol{q}) \dot{q}_{i} \dot{q}_{j}, \quad k=1, \ldots, n$

$\Gamma_{k i j}(\boldsymbol{q})=\frac{1}{2}\left(\frac{\partial A_{i j}}{\partial q_{k}}-\frac{\partial A_{k j}}{\partial q_{i}}-\frac{\partial A_{k i}}{\partial q_{j}}\right)$.

The problem is to find a feedback control $\boldsymbol{Q}(\boldsymbol{q}, \dot{\boldsymbol{q}})$ satisfying (2) and bringing the system (1) (or (4)) from any given initial state

$\boldsymbol{q}\left(t_{0}\right)=\mathbf{q}^{0}, \quad \dot{\boldsymbol{q}}\left(t_{0}\right)=\dot{\mathbf{q}}^{0}$ 
to the prescribed terminal state

$\boldsymbol{q}\left(t_{1}\right)=\mathbf{q}^{1}, \quad \dot{\boldsymbol{q}}\left(t_{1}\right)=0$

in finite time. Here, the instant $t_{0}$ and the vectors $\mathbf{q}^{0}, \dot{q}^{0}$ and $\mathbf{q}^{1}$ are fixed whereas $t_{1}$ is free. The case of nonzero terminal velocity $\dot{\mathbf{q}}\left(t_{1}\right) \neq 0$ was considered in (Ananyevskii et al, 1995). We shall discuss two approaches to the control problem stated above.

\section{THE FIRST APPROACH}

Multiplying (4) by the matrix $\boldsymbol{A}\left(\mathbf{q}_{1}\right) \boldsymbol{A}^{-1}(\boldsymbol{q})$, we get

$$
\begin{aligned}
& \mathbf{A}_{1} \ddot{\boldsymbol{q}}=\boldsymbol{Q}+\boldsymbol{R}(\boldsymbol{q}, \dot{\boldsymbol{q}}, t, \boldsymbol{Q}), \quad \mathbf{A}_{1}=\boldsymbol{A}\left(\boldsymbol{q}_{1}\right) \\
& \boldsymbol{R}=\mathbf{A}_{1} \boldsymbol{A}^{-1}(\boldsymbol{q})(\boldsymbol{F}+\boldsymbol{S})+\mathbf{A}_{1} \boldsymbol{A}^{-1}(\boldsymbol{q}) \boldsymbol{Q}-\boldsymbol{Q}
\end{aligned}
$$

Suppose all motions under consideration as well as the initial and terminal states (6) and (7) lie in some domain $W$ of the $2 n$-space $(\boldsymbol{q}, \dot{\boldsymbol{q}})$ where

$$
\left|R_{k}\right| \leq R_{k}^{0}<Q_{k}^{0}, \quad k=1, \ldots, n
$$

for all $t \geq t_{0},(\boldsymbol{q}, \dot{\boldsymbol{q}}) \in W$ and $Q_{k}$ satisfying (2). Here $R_{k}^{0}$ are constants. The inequalities (9) can be verified by means of the following Lemma given in (Chernousko, 1990, 1993a, 1993b).

Lemma Suppose the following inequalities

$$
\begin{aligned}
& \left\|A_{1} z\right\| \geq \mu_{1}\|z\|, \quad\left\|\left[A(q)-A_{1}\right] z\right\| \leq \mu\|z\|, \\
& \left|F_{k}+S_{k}\right| \leq \nu Q_{k}^{0}, \quad k=1, \ldots, n, \quad 0<\mu<\mu_{1}, \nu>0
\end{aligned}
$$

hold for any $z \in R^{n}$, all $t \geq t_{0}$, and all $(q, \dot{q}) \in W$ where $\mu_{1}, \mu$, and $\nu$ are constants. Then for all $t \geq t_{0}$, all $(q, \dot{q}) \in W$, and all $Q$ satisfying (2) we have

$$
\begin{aligned}
\left|R_{k}\right| & \leq \nu Q_{k}^{0}+\mu\left(\mu_{1}-\mu\right)^{-1}(1+\nu) Q^{0}, \\
Q^{0} & =\left[\sum_{k=1}^{n}\left(Q_{k}^{0}\right)^{2}\right]^{1 / 2}, \quad k=1, \ldots, n .
\end{aligned}
$$

It follows from the Lemma that the inequalities (9) are satisfied, if $\boldsymbol{A}(\boldsymbol{q})$ is close to $\mathbf{A}_{\mathbf{1}}$ and $Q_{k}^{0}$ are sufficiently large. By the change of variables $\mathbf{A}_{\mathbf{1}}\left(\boldsymbol{q}-\mathbf{q}_{1}\right)=\boldsymbol{y}$ we reduce the system (8), constraints (2) and (9), and terminal conditions ( 7 ) to the form

$$
\begin{aligned}
& \ddot{y}_{k}=Q_{k}+R_{k}, \quad\left|Q_{k}\right| \leq Q_{k}^{0}, \quad\left|R_{k}\right| \leq R_{k}^{0}<Q_{k}^{0}, \\
& y_{k}\left(t_{1}\right)=\dot{y}_{k}\left(t_{1}\right)=0
\end{aligned}
$$


We regard the terms $R_{k}$ in (10) as independent and arbitrary uncertain disturbances subject to the constraints (9). Applying the approach of differential games, we can treat (10) as a set of $n$ separate independent subsystems with one degree of freedom each. Here, $Q_{k}$ and $R_{k}$ are the controls of the two players acting on the $k$ th subsystem. We shall find the feedback control $Q_{k}$ of the first player and thus obtain the solution of our control problem.

Using the change of variables

$y_{k}=Q_{k}^{0} x, Q_{k}=Q_{k}^{0} u, R_{k}=Q_{k}^{0} v, \rho=R_{k}^{0} / Q_{k}^{0}<1$,

we reduce the $k$ th subsystem (10) to the normalized form

$\ddot{x}=u+v,|u| \leq 1,|v| \leq \rho<1, x(\tau)=\dot{x}(\tau)=0$

where $\tau$ is the terminal time.

Let us find the feedback control $u(x, \dot{x})$ as a time-optimal control of the first player in the differential game (12). This linear differential game is reduced (Krasovskii, 1970) to the time-optimal control problem for the system

$\ddot{x}=(1-\rho) u,|u| \leq 1, x(\tau)=\dot{x}(\tau)=0, \tau \rightarrow \min$

obtained from (12) through replacing $v$ by $(-\rho u)$. The optimal feedback control for the system (13) is (Pontryagin et al, 1972)

$$
\begin{aligned}
u(x, \dot{x}) & =\operatorname{sign} \psi_{\rho}(x, \dot{x}) \quad \text { if } \quad \psi_{\rho} \neq 0, \\
u(x, \dot{x}) & =\operatorname{sign} x=-\operatorname{sign} \dot{x} \quad \text { if } \quad \psi_{\rho}=0, \\
\psi_{\rho}(x, \dot{x}) & =-x-\dot{x}|\dot{x}|[2(1-\rho)]^{-1}
\end{aligned}
$$

where $\psi_{\rho}$ is the switching function. Returning to the original variables using $\boldsymbol{y}=\mathbf{A}_{1}\left(\boldsymbol{q}-\mathbf{q}_{1}\right)$ and (13), we obtain the desired feedback control as follows

$Q_{k}(\boldsymbol{q}, \dot{\boldsymbol{q}})=Q_{k}^{0} u(x, \dot{x}), \quad x=\left(Q_{k}^{0}\right)^{-1}\left[\mathbf{A}_{1}\left(\boldsymbol{q}-\mathbf{q}_{1}\right)\right]_{k}$,

$\dot{x}=\left(Q_{k}^{0}\right)^{-1}\left(\mathbf{A}_{1} \dot{\boldsymbol{q}}\right)_{k}$

where $u(x, \dot{x})$ is defined in (14) and $\rho$ is given by (11) for each $k$. The total time of control is finite and does not exceed the maximum (over all $k$ ) of the control times for the subsystems (13). We have the following upper estimate for the terminal instant $t_{1}$ :

$$
\begin{aligned}
& t_{1}-t_{0} \leq \max _{k} \tau\left(x_{k}^{0}, \dot{x}_{k}^{0}\right), \quad k=1, \ldots, n, \\
& x_{k}^{0}=\left(Q_{k}^{0}\right)^{-1}\left[\mathbf{A}_{1}\left(\mathbf{q}^{0}-\mathbf{q}^{1}\right)\right]_{k}, \quad \dot{x}_{k}^{0}=\left(Q_{k}^{0}\right)^{-1}\left(\mathbf{A}_{1} \mathbf{q}^{0}\right)_{k}, \\
& \tau(\xi, \eta)=(1-\rho)^{-1}\left\{\left[2 \eta^{2}-4(1-\rho) \xi \gamma\right]^{1 / 2}-\eta \gamma\right\} \\
& \gamma=\operatorname{sign} \psi_{\rho}(\xi, \eta), \quad \rho=R_{k}^{0} / Q_{k}^{0}<1 .
\end{aligned}
$$




\section{SOME GENERALIZATIONS}

The approach described above consists of two stages: 1) decomposition of the original system into simple subsystems; 2) design of the feedback control for the subsystem. Both stages can be modified.

At the first stage, the system (4) can be reduced to the set of subsystems other than (13). Namely, the following subsystems with linear or nonlinear damping can be used

$\ddot{x}+\lambda \dot{x}=u+v, \lambda=$ const $>0 ; \quad \ddot{x}+f(\dot{x})=u+v$

where $u$ and $v$ are again the control and disturbance, respectively. Here the smooth function $f(z)$ has the following properties:

$z f(z)>0, \quad f^{\prime}(z)>0 \quad$ if $\quad z \neq 0, \quad f(0)=0$.

Some results for equations (16) are presented in (Chernousko, 1993a, 1993b).

At the second stage, the game approach can be replaced by other methods for control design. The simplest possibility is to ignore the disturbance $v$ while designing the control $u$ and then to use thus obtained control for the system subject to the disturbance. Applying this simplified approach to the system (12), we should take the control $u(x, \dot{x})$ in the form (14) with $\rho=0$. The analysis presented in (Chernousko 1990, 1993a, 1993b) shows that the dynamics of the system (14) under this simplified control depends essentially on the parameter $\rho$, the critical value of $\rho$ being equal to the golden section ratio

$\rho^{*}=\left(5^{1 / 2}-1\right) / 2=0.618 \ldots$

If $\rho<\rho^{*}$, then the system (12) under the simplified control and arbitrary disturbance $v,|v| \leq \rho$, reaches the terminal state $x=\dot{x}=0$ in finite time. If $\rho=\rho^{*}$, then the system stays in the bounded domain of the phase plane, and there exists an admissible disturbance such that the system has a periodic solution and never reaches the terminal state. If $\rho>\rho^{*}$, then there exist admissible disturbances such that the system (12) has unbounded solutions: $x \rightarrow \infty$ as $t \rightarrow \infty$. Hence, our system (12) under the simplified control is controllable, if and only if $\rho<\rho^{*}$, whereas under the game control it is controllable, if and only if $\rho<1$. The same conclusion is true also for the systems (16). Hence, the bounded disturbance can be ignored while designing the control, if the ratio of the amplitudes of the disturbance and control is less than the golden section ratio $\rho^{*}$. Obviously, the game approach is preferable to the simplified one.

\section{THE SECOND APPROACH}

We consider again the system (1) (or (4)) and assume that all its motions under investigation belong to the set $D$ of the $\boldsymbol{q}$-space. The set $D$ may coincide with the whole space $R^{n}$. Suppose that

$m\|\mathbf{z}\|^{2} \leq(\boldsymbol{A}(\boldsymbol{q}) \mathbf{z}, \mathbf{z}) \leq M\|\mathbf{z}\|^{2}, \quad 0<m<M$,

$\left|\partial A_{i j} / \partial q_{k}\right| \leq C, \quad i, j, k=1, \ldots, n$. 
for any $\mathbf{z} \in R^{n}$ and all $\boldsymbol{q} \in D$. Here $m, M$, and $C$ are positive constants. Let the uncontrollable forces $\boldsymbol{F}$ in (1) and (4) consist of two terms

$\boldsymbol{F}=\boldsymbol{G}+\boldsymbol{\Phi}, \quad \boldsymbol{G}=\left(G_{1}, \ldots, G_{n}\right), \quad \boldsymbol{\Phi}=\left(\lambda_{1}, \ldots, \lambda_{n}\right)$

subjected to different constraints.

The dissipative forces $\boldsymbol{G}(\boldsymbol{q}, \dot{\boldsymbol{q}}, t)$ satisfy two conditions:

1) $(\boldsymbol{G}, \dot{\boldsymbol{q}}) \leq 0$ for all $\boldsymbol{q} \in D$, and all $t \geq t_{0}$;

2) $\left|G_{k}\right| \leq G_{k}^{0}(\alpha), k=1, \ldots, n$, if $\left|\dot{q}_{i}\right| \leq \alpha<\varepsilon_{0}$ for all $i=1, \ldots, n$.

Here $\varepsilon_{0}>0$ is a positive number, $G_{k}^{0}(\alpha)$ are continuous monotone increasing functions for $\alpha \in\left[0, \varepsilon_{0}\right]$, and $G_{k}^{0}(\alpha)=0, k=1, \ldots, n$.

The forces $\Phi$ in (18) are arbitrary but bounded disturbances such that

$\left|\lambda_{k}\right| \leq \lambda_{k}^{0}<Q_{k}^{0}, \quad i=1, \ldots, n$

where $\lambda_{k}^{0}>0$ are given constants.

Let us introduce the sets in the $2 n$-space $(q, \dot{q})$

$\Omega_{1}=\left\{(\boldsymbol{q}, \dot{\boldsymbol{q}}): \boldsymbol{q} \in D ; \exists k,\left|\dot{q}_{k}\right|>\varepsilon\right\}$,

$\Omega_{2}=\left\{(\boldsymbol{q}, \dot{\boldsymbol{q}}): \boldsymbol{q} \in D ; \forall k,\left|\dot{q}_{k}\right| \leq \varepsilon\right\}$

The number $\varepsilon>0$ will be chosen later. We define our feedback control in $\Omega_{1}$ as follows

$Q_{k}(\boldsymbol{q}, \dot{\boldsymbol{q}})=-Q_{k}^{0} \operatorname{sign} \dot{q}_{k} \quad$ if $\dot{q}_{k} \neq 0$,

$Q_{k}(\boldsymbol{q}, \dot{\boldsymbol{q}})=0 \quad$ if $\dot{q}_{k}=0, k=1, \ldots, n$.

By taking into account equations (18), (21), and the assumptions made, we obtain from (4)

$d T / d t \leq(\boldsymbol{Q}+\boldsymbol{\Phi}, \dot{\boldsymbol{q}}) \leq-\sum_{k=1}^{n}\left(Q_{k}^{0}-\lambda_{k}^{0}\right)\left|\dot{q}_{k}\right| \leq-r|\dot{\boldsymbol{q}}|$,

$r=\min _{k}\left(Q_{k}^{0}-\lambda_{k}^{0}\right)>0, \quad k=1, \ldots, n$.

The upper estimate (17) for $T$ implies that $|\dot{\boldsymbol{q}}| \geq(2 T / M)^{1 / 2}$. Hence, we have in $\Omega_{1}$ :

$2 T^{1 / 2} d T^{1 / 2} / d t \leq-r(2 T / M)^{1 / 2}, \quad$ or $\quad d T^{1 / 2} / d t \leq-r(2 M)^{-1 / 2}$.

Integrating the latter inequality, we obtain that, if the motion starts in $\Omega_{1}$, then the control (21) steers our system (4) to the boundary between $\Omega_{1}$ and $\Omega_{2}$ in finite time. The system reaches this boundary at the instant $t_{*}$ such that

$t_{*}-t_{0} \leq(2 M)^{1 / 2} r^{-1}\left[T_{0}^{1 / 2}-(m / 2)^{1 / 2} \varepsilon\right], \quad T_{0}=\left(A\left(\mathbf{q}^{0}\right) \dot{\mathbf{q}}^{0}, \dot{\mathbf{q}}^{0}\right) / 2$. 
In $\Omega_{2}$, we rewrite equation (4) as follows

$\ddot{q}=U+V, U=A^{-1} Q, V=A^{-1}(G+\Phi+S)$.

Let us regard $U$ and $V$ as a control and disturbance, respectively. It follows from (17)-(20) that

$$
\begin{aligned}
& \left|V_{k}\right| \leq V^{0}, V^{0}=m^{-1}\left[G^{0}(\varepsilon)+\lambda^{0}+1.5 C n^{5 / 2} \varepsilon^{2}\right], \\
& G_{k}^{0}(\varepsilon)=\left\{\sum_{k=1}^{n}\left[G_{k}^{0}(\varepsilon)\right]^{2}\right\}^{1 / 2}, \quad \lambda^{0}=\left[\sum_{k=1}^{n}\left(\lambda_{k}^{0}\right)^{2}\right]^{1 / 2} .
\end{aligned}
$$

We impose the following constraints on $\boldsymbol{U}$

$$
\left|U_{k}\right| \leq U^{0}, U^{0}=r M^{-1} n^{-1 / 2}, r=\min _{k} Q_{k}, k=1, \ldots, n .
$$

It follows from (17), (22), and (24) that

$\boldsymbol{Q}=\boldsymbol{A} U,\left|Q_{k}\right| \leq\|\boldsymbol{A U}\| \leq M\|\boldsymbol{U}\| \leq M n^{1 / 2} U^{0}=r \leq Q_{k}^{0}$.

Hence, if the inequalities (24) hold, our original constraints (2) are satisfied. Equations (22) and constraints (23) and (24) can be rewritten in the form

$\ddot{q}_{k}=U_{k}+V_{k},\left|U_{k}\right| \leq U^{0},\left|V_{k}\right| \leq V^{0}, k=1, \ldots, n$

quite similar to (12). The only difference is that the solution of the system (25) must satisfy the state constraint

$\left|\dot{q}_{k}(t)\right| \leq \varepsilon, \quad k=1, \ldots, n$

following from the requirement that our system should not leave the set $\Omega_{2}$ from (20). The feedback control satisfying this additional condition can be obtained as a modification of (14), if $\rho=V^{0} / U^{0}<1$. We take

$$
\begin{aligned}
U_{k}\left(q_{k}, \dot{q}_{k}\right)= & U^{0} \operatorname{sign}\left[\psi\left(x_{k}\right)-\dot{q}_{k}\right] \quad \text { if } \quad \dot{q}_{k} \neq \psi\left(x_{k}\right), \\
U_{k}\left(q_{k}, \dot{q}_{k}\right)= & U^{0} \operatorname{sign} x_{k}=-U^{0} \operatorname{sign} \dot{q}_{k} \quad \text { if } \quad \dot{q}_{k}=\psi\left(x_{k}\right) ; \\
\psi(x)= & -\left[2 U^{0}(1-\rho)|x|\right]^{1 / 2} \operatorname{sign} x \quad \text { if } \quad|x| \leq x^{*}, \\
\psi(x)= & -\delta \operatorname{sign} x \quad \text { if }|x| \geq x^{*} ; x_{k}=q_{k}-q_{k}^{1}, x^{*}=\delta^{2}\left[2 U^{0}(1-\rho)\right]^{-1}, \\
& \delta \in(0, \varepsilon), \rho=V^{0} / U^{0}<1, k=1, \ldots, n .
\end{aligned}
$$

Here, $\delta$ is an arbitrary number from the interval $(0, \varepsilon)$, and $x^{*}$ is chosen so that the function $\psi(x)$ is continuous. The switching curve $\dot{q}_{k}=\psi\left(x_{k}\right)$ defined by (27) is symmetrical with respect to the point $x_{k}=\dot{q}_{k}=0$, lies in the band (26), and consists of two arcs of parabolas for $\left|x_{k}\right| \leq x^{*}$ and two rays $\dot{q}_{k}= \pm \delta$ for $\left|x_{k}\right| \geq x^{*}$. As shown in (Chernousko, 
$1992 \mathrm{a}, 1993 \mathrm{~b})$, if $\rho<1$, the feedback control (27) brings the system (25) to the terminal state (7) in finite time under any admissible $V_{k},\left|V_{k}\right| \leq V^{0}$, and the constraint (26) is satisfied. To ensure the condition $\rho<1$, we should choose $\varepsilon$ so that

$\rho=V^{0} / U^{0}=(M / m) n^{1 / 2} r^{-1}\left[G^{0}(\varepsilon)+\lambda^{0}+1.5 C n^{5 / 2} \varepsilon^{2}\right]<1$.

Since $G^{0}(\varepsilon) \rightarrow 0$ as $\varepsilon \rightarrow 0$, the condition (28) can be satisfied, if and only if

$\lambda^{0}<(m / M) r n^{-1 / 2}$.

Hence, if (29) holds, our control problem is solvable by the second approach described in this section. The condition (29) is always satisfied, if the disturbances are absent $(\boldsymbol{\Phi}=0)$.

Let (29) hold. Then the parameter $\varepsilon$ in (20) should be chosen so that $\varepsilon \leq \varepsilon_{0}$ and (28) holds. Then the feedback control $\boldsymbol{Q}(\boldsymbol{q}, \dot{\boldsymbol{q}})$, defined by (21) in $\Omega_{1}$ and by $\boldsymbol{Q}=\boldsymbol{A}(\boldsymbol{q}) \boldsymbol{U}$ and (27) in $\Omega_{2}$, brings our system (4) from any initial state (6) to the prescribed terminal state (7) in finite time under any admissible disturbances. The upper estimate on the total time of motion is given by

$$
\begin{aligned}
& t_{1}-t_{0} \leq \delta^{-1} \max _{k}\left|q_{k}^{0}-q_{k}^{1}\right|+(2 M)^{1 / 2} r^{-1}\left[T_{0}^{1 / 2}-(m / 2)^{1 / 2} \varepsilon\right] \\
&+(M / m)^{1 / 2} r^{-1} \delta^{-1}\left(T_{0}-m \varepsilon^{2} / 2\right) \\
&+\left(2 \varepsilon^{2}+4 \varepsilon \delta+3 \delta^{2}\right) \delta^{-1}\left[2 U^{0}(1-\rho)\right]^{-1}, \quad k=1, \ldots, n, \\
& \rho=V^{0} / U^{0}, \quad \delta \in(0, \varepsilon) .
\end{aligned}
$$

Note that the parameter $\delta$ should be taken close to $\varepsilon$, because the greater is $\delta$, the smaller is the time of motion.

\section{DISCUSSION}

Let us compare the two approaches described in Sections 3 and 5. The first approach is based on the assumptions imposed in the $2 n$-dimensional $(\boldsymbol{q}, \dot{\boldsymbol{q}})$-space, whereas the second one deals with assumptions in the $n$-dimensional $\boldsymbol{q}$-space. Hence, the assumptions of the second approach are less restrictive. On the other hand, the second approach incorporates motions with low velocities in the set $\Omega_{2}$ and, hence, the total time of motion may be greater for the second approach.

Both approaches do not presume that the external forces are known. These forces may be uncertain, with only the bounds on them being essential.

Equations (1) or (4) are typical for robotic manipulators. Here, $q_{k}$ are generalized coordinates of a robot (angles of rotation and linear displacements for revolute and prismatic joints, respectively), whereas $Q_{k}$ are control torques, forces, or electric voltages. Computer simulation of the proposed control laws for robots with different kinematics showed quite satisfactory results. 


\section{DISTRIBUTED PARAMETERS SYSTEMS}

Let us consider briefly the decomposition of controlled distributed parameters systems described by linear partial differential equations of one of the two following types

$$
\begin{aligned}
w_{t} & =A w+v \\
w_{t t} & =A w+v
\end{aligned}
$$

Here $w(\boldsymbol{x}, t)$ is a scalar state depending on time $t$ and $n$-vector $\boldsymbol{x}, v$ is a scalar control, and $A$ is a linear selfadjoint differential operator of the even order $2 m$ (in $\boldsymbol{x}$ ) independent of $t$. For example, if $m=1, A=\Delta$, then (30) is a parabolic equation, whereas (31) is a wave equation; if $m=2, A=-\triangle^{2}$, then (31) describes elastic vibrations of a beam $(n=1)$ or a plate $(n=2)$. Equations $(30),(31)$ are considered in the domain $x \in \Omega$, $t \geq 0$ under the homogeneous boundary conditions

$$
M w=0, \quad M=\left(M_{1}, \ldots, M_{m}\right), \quad \boldsymbol{x} \in \Gamma=\partial \Omega
$$

where $M_{j}$ is a linear differential operator of the order less than $2 m$ with coefficients independent of $t$. The initial conditions for equation (31) are

$w(\boldsymbol{x}, 0)=w_{0}(\boldsymbol{x}), \quad w_{t}(\boldsymbol{x}, 0)=w_{t 0}(\boldsymbol{x}), \quad \boldsymbol{x} \in \Omega$,

whereas in case of (30) the second condition (33) should be omitted. The control $v$ in (30) and (31) is subject to the constraint

$|v(\boldsymbol{x}, t)| \leq v^{0}, \quad \boldsymbol{x} \in \Omega, \quad t \geq 0$

where $v^{0}>0$ is a given constant.

We are to find the control $v$ satisfying (34) and such that the corresponding solution of the initial boundary value problem described by (31)-(33) becomes zero at $t=T$ : $w(\boldsymbol{x}, T)=w_{t}(\boldsymbol{x}, T)=0$. For equation (30), one should omit the latter equality and the second condition (33). The time $T$ is not fixed a priori.

Let us consider the eigenvalue problem

$A \varphi=-\lambda \varphi, \quad x \in \Omega ; \quad M \varphi=0, \quad x \in \Gamma$

and assume that all eigenvalues $\lambda_{k}>0, k=1,2, \ldots$, and $\lambda_{1} \leq \lambda_{2} \leq \ldots$. If there is also a zero eigenvalue, we put $\lambda_{0}=0$. We present the state and control as series in orthonormal eigenfunctions $\varphi_{k}$

$w(\boldsymbol{x}, t)=\sum q_{k}(t) \varphi_{k}(\boldsymbol{x}), \quad v(\boldsymbol{x}, t)=\sum u_{k}(t) \varphi_{k}(\boldsymbol{x})$

Substituting (36) into (30) and (31) and using (35), we obtain the respective equations for $\boldsymbol{q}_{k}$

$\dot{q}_{k}+\lambda_{k} q_{k}=u_{k}, \quad \ddot{q}_{k}+\lambda_{k} q_{k}=u_{k}, \quad \lambda_{k} \geq 0$. 
The initial conditions (33) are replaced by

$q_{k}(0)=\int_{\Omega} w_{0} \varphi_{k} d x, \quad \dot{q}_{k}(0)=\int_{\Omega} w_{t 0} \varphi_{k} d x$

Let us impose the constraints on controls $u_{k}$ :

$\left|u_{k}\right| \leq U_{k}, \quad t \geq 0$.

The bounds $U_{k}>0$ should be chosen so that the constraint (34) holds. It is sufficient to require that

$\sum U_{k} N_{k} \leq v^{0}, \quad N_{k}=\max _{\boldsymbol{x} \in \Omega}\left|\varphi_{k}(\boldsymbol{x})\right|$.

Since each equation (37) is controllable, we can choose the time-optimal control $u_{k}$ bringing the $k$ th mode to the zero terminal state under the constraint (39). For the first-order equations (37), we have

$$
\begin{array}{rlrl}
u_{k}\left(q_{k}\right) & =-U_{k} \operatorname{sign} q_{k}, \quad q_{k} \neq 0, & u_{k}(0)=0 \\
T_{k} & =\lambda_{k}^{-1} \ln \left[1+\lambda_{k}\left|q_{k}(0)\right| U_{k}^{-1}\right], & & \lambda_{k}>0, \\
T_{0} & =\left|q_{0}(0)\right| U_{0}^{-1}, \quad \lambda_{0}=0 &
\end{array}
$$

where $T_{k}$ is the minimal time for the $k$ th mode. To minimize the total time of motion $T=\max _{k} T_{k}$, we should choose $U_{k}$ in such a way that all $T_{k}$ are equal: $T_{k}=T, k \geq 0$. We obtain the following condition on $T$

$$
\sum \lambda_{k}\left[\exp \left(\lambda_{k} T\right)-1\right]^{-1}\left|q_{k}(0)\right| N_{k} \leq v^{0} .
$$

It can be shown that the series (42) converges for all $T>0$, and its sum decreases monotonically from $\infty$ to 0 as $T$ changes from 0 to $\infty$. Hence, there exists $T$ such that (42) holds, and our control problem is solved by the proposed method. The control for each $k$ th mode is given in an explicit form.

For the second-order equations (37), the situation is more complicated. The feedback time-optimal control $u_{k}\left(q_{k}, \dot{q}_{k}\right)$ for each subsystem (37) under the constraints (39) is again given in an explicit form (Pontryagin et al, 1972), but the corresponding minimal times $T_{k}$ àre not expressed explicitly. We have obtained upper estimates on these times and used them to derive sufficient controllability conditions for the system (31). These conditions can be presented as convergence conditions for certain series (Chernousko, 1992b). For example, if the following two series

$$
\sum \lambda_{k}^{1 / 2}\left|q_{k}(0)\right| N_{k}<\infty, \quad \sum\left|\dot{q}_{k}(0)\right| N_{k}<\infty
$$

converge, then the system (31) is controllable by the proposed method.

Using (43), sufficient controllability conditions for various specific cases were obtained, including control of elastic systems (membranes, beams, and plates of various shapes under different boundary conditions) by means of bounded distributed forces. 
For example, let us consider vibrations of an elastic beam controlled by distributed forces. Equation (31) and the control constraint (34) can be presented as follows

$w_{t t}=-w_{x x x x}+v, \quad|v| \leq v^{0}, \quad a \leq x \leq b$.

The boundary conditions for the case of hinged support at the both ends of the beam are $w=w_{x x}=0$ for $x=a, x=b$. It can be shown that the both series (43) converge, if the initial distributions of elastic displacements $w_{0}(x)=w(x, 0)$ and velocities $w_{t 0}(x)=w_{t}(x, 0)$ of the beam satisfy the following smoothness conditions:

$w_{0} \in C^{4}, \quad w_{t 0} \in C^{2}, \quad x \in[a, b]$.

\section{CONCLUSION}

The results presented above are based on the decomposition of the original system into simple subsystems and use optimal control methods. Since the time of motion is minimized for each subsystem, these methods may be regarded as suboptimal. The total time of motion is finite: the motion stops when the last degree of freedom reaches its terminal state. Upper estimates on terminal times are given.

\section{ACKNOWLEDGEMENTS}

The paper is based on research sponsored by the International Science Foundation, Grant M4F000, and by the Russian Foundation of Basic Research, Project 93-01-16286.

\section{REFERENCES}

Ananyevskii, I.M., Dobrynina, I.S. and Chernousko, F.L. (1995) Method of decomposition in the control problem for a mechanical system. Izvestiya of the Russian Academy of Sciences, Control Theory and Systems, 2, 3-14.

Chernousko, F.L. (1990) Decomposition and suboptimal control in dynamical systems. Journal of Applied Mathematics and Mechanics (PMM), 54, 6, 727-34.

Chernousko, F.L. (1992a) Control synthesis in a non-linear dynamical system. Journal of Applied Mathematics and Mechanics (PMM), 56, 2, 157-66.

Chernousko, F.L. (1992b) Bounded controls in distributed-parameter systems. Journal of Applied Mathematics and Mechanics (PMM), 56, 5, 707-23.

Chernousko, F.L. (1993a) Decomposition and suboptimal control in dynamic systems. Optimal Control Applications and Methods, 14, 2, 125-43.

Chernousko, F.L. (1993b) The Decomposition of Controlled Dynamic Systems, in Advances in Nonlinear Dynamics and Control: A Report from Russia (ed. A.B. Kurzhanski), Birkhäuser, Boston.

Krasovskii, N.N. (1970) Game Problems of the Encounter of Motions. Nauka, Moscow.

Pontryagin, L.S., Boltyansky, V.G., Gamkrelidze, R.V. and Mishchenko, E.F. (1972) Mathematical Theory of Optimal Processes. Wiley-Interscience, New York. 